\title{
Electron Bessel beam diffraction for precise and accurate nanoscale strain mapping $\odot \circ$
}

\author{
Cite as: Appl. Phys. Lett. 114, 243501 (2019); doi: 10.1063/1.5096245 \\ Submitted: 15 March 2019 - Accepted: 23 May 2019 . \\ Published Online: 17 June 2019
}

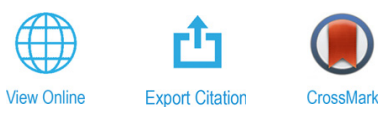

\begin{abstract}
Giulio Guzzinati, ${ }^{1, a)}$ (D) Wannes Ghielens, ${ }^{2}$ Christoph Mahr, ${ }^{3,4}$ (D) Armand Béché, ${ }^{7}$ (D) Andreas Rosenauer, ${ }^{3,4}$ (D) Toon Calders, ${ }^{2}$ (iD and Jo Verbeeck
\end{abstract}

\author{
AFFILIATIONS \\ ${ }^{7} E M A T$, University of Antwerp, Groenenborgerlaan 171, 2020 Antwerp, Belgium \\ ${ }^{2}$ Adrem Data Lab, University of Antwerp, Middelheimlaan 1, 2020 Antwerp, Belgium \\ ${ }^{3}$ Institute of Solid State Physics, University of Bremen, Otto-Hahn-Allee 1, D-28359 Bremen, Germany \\ ${ }^{4}$ MAPEX Center for Materials and Processes, University of Bremen, Bibliothekstr. 1, D-28359 Bremen, Germany

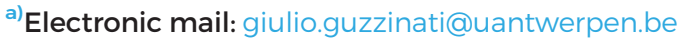

\begin{abstract}
Strain has a strong effect on the properties of materials and the performance of electronic devices. Their ever shrinking size translates into a constant demand for accurate and precise measurement methods with a very high spatial resolution. In this regard, transmission electron microscopes are key instruments thanks to their ability to map strain with a subnanometer resolution. Here, we present a method to measure strain at the nanometer scale based on the diffraction of electron Bessel beams. We demonstrate that our method offers a strain sensitivity better than $2.5 \times 10^{-4}$ and an accuracy of $1.5 \times 10^{-3}$, competing with, or outperforming, the best existing methods with a simple and easy to use experimental setup.
\end{abstract}

Published under license by AIP Publishing. https://doi.org/10.1063/1.5096245

Strain in materials is of extreme importance in a wide variety of technological applications and particularly in nanoelectronics where, besides impacting the devices' lifetime and performance, it is used deliberately for engineering the electronic transport properties. ${ }^{1,2}$ The ever shrinking size of electronic devices translates into an increasing demand for accurate and precise strain measurement methods with a very high spatial resolution.

When engineering nanomaterials, characterizing nanostructures or current generation nanoelectronic devices, the spatial resolution becomes paramount. Techniques such as X-ray diffraction or $\mu$-Raman spectroscopy are quite easily accessible methods and offer very good strain sensitivity [respectively, $\sigma=1 \times 10^{-5}$ (Ref. 3) and $\sigma$ $=1 \times 10^{-4}$ (Ref. 4)] but a spatial resolution too low to investigate strain within a single device. ${ }^{5}$ Transmission electron microscopy (TEM) offers strain measurements with the highest spatial resolution, and many methods have been developed or adapted to this purpose. Strain can be measured through convergent beam electron diffraction (CBED), high resolution conventional and scanning TEM (HRTEM and HRSTEM) imaging including Moiré fringe analysis (Moiré fringes appear when using a HRSTEM setup to scan at a lower magnification, due to the low-frequency sampling of the crystal lattice) as well as nanobeam electron diffraction (NBED), which can also be performed in conjunction with precession electron diffraction (N-PED) ${ }^{6-10}$ Of these techniques, the best accuracy and precision are offered by nanobeam precession electron diffraction with a spatial resolution better than $1 \mathrm{~nm}$, a strain sensitivity of $\sigma=2 \times 10^{-4}$, and an accuracy of $\Delta$ $=1 \times 10^{-3}$ although it requires additional specialized hardware. ${ }^{11,12}$ Conventional nanobeam electron diffraction is also a highly precise $\left(\sigma=6 \times 10^{-4}\right)$ and accurate $\left(\Delta=2 \times 10^{-3}\right)$ technique although it is not on a par with N-PED and suffers the same limits in terms of resolution.

Here, we propose a diffraction-based strain measurement protocol which can be applied on unmodified transmission electron microscopes by just replacing an aperture of the illumination system. It offers a simplified setup and performances approaching those of $\mathrm{N}$ PED and clearly outperforming NBED, while providing an excellent spatial resolution.

A fundamental problem with electron diffraction experiments is dynamical scattering. Due to the very strong interaction between the electron beam and the sample, the electrons are scattered many times within the specimen, leading to several unwanted effects, from nonlinearities in the intensity of the diffracted beams to a nonhomogeneous shape of the diffracted disks, complicating significantly the extraction of structural information. ${ }^{13}$ Precession electron diffraction mitigates 
these problems by varying the beam's incident direction. ${ }^{14}$ By entering the sample under a shallow angle (below $2^{\circ}$, but below $0.5^{\circ}$ for strain applications) with respect to one of the main crystallographic directions, the electrons are scattered more weakly, and therefore, multiple scattering becomes less common. While keeping the angle of incidence constant, the direction of incidence is varied azimuthally so that the pattern is averaged over the different configurations. This leads to quasikinematical diffraction patterns which are less sensitive to local sample variation (thickness, bending...) and are thus easier to interpret. ${ }^{14}$ This is typically achieved by using dedicated control hardware to rock the incident beam and then cancel this rocking after the interaction with the sample by using the microscope's deflecting coils so that the patterns for all different incident directions superimpose and are averaged, as schematically shown in Fig. 1(a). While the coils used are already part of the microscope, it is generally not possible or practical for the user to freely program them, making the purchase (or construction) of dedicated hardware necessary in most cases. This hardware then requires specific alignment procedures to get the two rocking processes to compensate each other as well as possible. This need for dedicated hardware and alignment has kept this powerful method from gaining widespread adoption.

Our proposal is inspired by precession, but rather than using different incidence directions in a sequential fashion, we realize a hollow-cone illumination where the rays from the different directions are present simultaneously. This creates a different type of diffraction pattern where each spot is replaced by a ring which, for typical precession angles, overlaps creating a more complex diffraction pattern (see Fig. 2), which required the development of a dedicated analysis protocol. In the following, we will test this method through computer simulations, then demonstrate it experimentally, and show that it offers performance close to those of N-PED without the extra

(a)

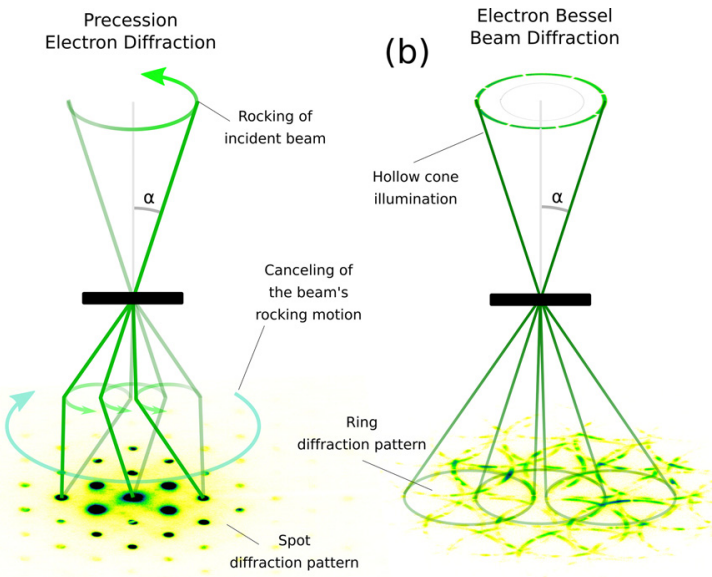

FIG. 1. Schematic representation of our proposed setup in relation to PED. (a) In precession electron diffraction, a low-convergence electron beam impinges on the sample under a fixed polar angle $\alpha$, while the azimuthal angle is rotated using deflectors. In order to still obtain a diffraction pattern formed of spots, another set of coils is used to cancel this rotation. The diffraction pattern is averaged over the different configurations, reducing dynamical effects. (b) In our proposal, we use an annular aperture to realize a hollow-cone illumination with semiconvergence angle $\alpha$ so that the rays from all the directions of incidence are simultaneously present. Due to this, the diffraction pattern is now constituted of rings, making it significantly harder to analyze. (a)

(c)
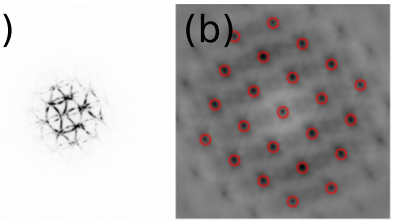

$\begin{array}{cccccc}(\mathrm{e}) & & 0 & & & \\ 0 & 0 & 0 & \\ 0 & 0 & & 0 & \\ 0 & 0 & 0 & 0 & 0 \\ 0 & 0 & 0 & 0 & 0 \\ 0 & 0 & 0 & 0 \\ & 0 & 0 & 0 \\ & & 0 & 0 & 0\end{array}$

FIG. 2. Ring diffraction patterns and analysis. (a) Ring diffraction pattern from the unstrained silicon substrate. (b) Autocorrelation function of the pattern, with marked positions for the maxima. (c) Ring diffraction pattern from a SiGe layer, significantly strained with respect to the substrate. (d) Autocorrelation function for the pattern in (c), also with marked peaks. (e) The extracted peaks differ visibly when overlaid. The strain is calculated by computing the affine transformation of the unstrained pattern that best allows us to overlap it with the strained one.

equipment, making this technique potentially useful to a much wider audience of electron microscopists and materials scientists.

The core idea behind this work was to implement a "parallel precession" experiment, which would have had a wide number of experimental advantages. To understand how our proposed setup is equivalent to precession, one can think of the ring diffraction pattern as a convergent beam diffraction pattern where one has removed the central part of the diffracted disks, as in Figs. 2(a) and 2 (c). In a diffraction pattern acquired with a parallel incident beam, the intensity of the diffracted spots depends on the exact direction of incidence and is very sensitive to even a slight tilt, in what is called the "rocking curve." In CBED, the diffraction pattern is formed of disks possessing nonhomogeneous intensity. Each point in the disk formed by the transmitted beam corresponds to a different direction of incidence and is associated with the corresponding diffracted points in the same position of the diffracted disks. The intensities of these points are identical to the intensities of the beams in a parallel-beam diffraction pattern with the same tilted direction of incidence. ${ }^{15,16}$ Keeping this in mind, it is clear that our approach yields exactly the same information as precession, as long as the rings do not overlap, i.e., as long as the semiconvergence angle $\alpha$ is lower than the Bragg angle for the sample under investigation. For overlapping rings, this is still largely valid, with the exception of the narrow overlap regions, where multibeam effects are possible and further complicate the interpretation. ${ }^{15}$

Similar diffraction patterns have also been realized before with a PED system by not "untilting" the beam in the projection system ${ }^{11,17}$ and have been proposed as a way to improve precision by increasing the area of each spot. ${ }^{17}$ Still, even in the simplest case of a nonoverlapping ring pattern, the reliable extraction of the ring positions has proven to be challenging. ${ }^{12}$

To explore the potential of our idea, we tested it using a simulated series of diffraction patterns. We performed multislice simulations with the STEMsim software ${ }^{18-21}$ using the same model structure as in Refs. 12, and 22-24. This structure is a Si-sample containing two embedded SiGe layers with different Ge-concentrations, of 38\% and $31 \%$, respectively; for details, see the supplementary material. 
While the resulting pattern is quite rich in detail, it is worth remembering that the strain measurement is a relatively simple problem. From all the wealth of details contained in this pattern, we seek only to measure the three in-plane strain components: the two normal strain components $\epsilon_{x x}$ and $\epsilon_{y y}$ and the shear strain $\epsilon_{x y}$, which are linked to the spacing between the Bragg reflections.

For this purpose, we based our analysis on the autocorrelation function. For a two-dimensional signal $f\left(\mathbf{q}_{\perp}\right)$, its autocorrelation function $\mathcal{A}_{f}\left(\mathbf{q}_{\perp}^{\prime}\right)$ is

$$
\begin{aligned}
\mathcal{A}_{f}\left(\mathbf{q}_{\perp}^{\prime}\right) & =\iint f\left(\mathbf{q}_{\perp}+\mathbf{q}_{\perp}^{\prime}\right) f^{\star}\left(\mathbf{q}_{\perp}\right) \mathrm{d} \mathbf{q}_{\perp} \\
& =\iint f\left(\mathbf{q}_{\perp}\right) f^{\star}\left(\mathbf{q}_{\perp}-\mathbf{q}_{\perp}^{\prime}\right) \mathrm{d} \mathbf{q}_{\perp},
\end{aligned}
$$

or using the Wiener-Khinchin theorem

$$
\mathcal{A}_{f}\left(\mathbf{q}_{\perp}^{\prime}\right)=\mathcal{F}^{-1}\left(\left|\mathcal{F}\left(f\left(\mathbf{q}_{\perp}\right)\right)\right|^{2}\right) .
$$

The autocorrelation function is essentially a measure of selfsimilarity, which is of similarity between different parts of the same signal (here, a diffraction pattern). For a signal containing a certain periodicity, the autocorrelation function will display peaks corresponding to the same period, as shown in Figs. 2(b) and 2(d). This means that we get a peak when the shift between the diffraction pattern and its copy is such that the $(0,0,0)$ ring in the copy overlaps a Bragg diffracted ring, e.g., the $(0,0,2)$ in the original pattern and the opposite $(0,0,-2)$ diffracted ring overlaps with the $(0,0,0)$ ring of the original pattern. This superposition and integration of the ring intensities, in our approach, have played a role reminiscent of the derocking procedure in conventional precession, averaging over the different parts of the rocking curve. It is also worth noting that in every circumstance, the autocorrelation function is always centered (i.e., the overlap between two copies of the pattern is the highest with no shift) and is centrosymmetric [as shown in Eq. (1)], which simplifies its analysis.

The peaks appear to be small and sitting over a large background, which is due to the fact that even when the rings of the copies of the pattern are not perfectly aligned, the overlap is still significant. To remove its effect, we normalize the contrast radially by fitting the background's strength to a polynomial, then locate the peaks, and fit their shape to a high-order polynomial surface, from which the peak's position is extracted with subpixel precision. Once these positions have been extracted, the problem is reduced to finding the affine transform that best overlaps them with those from a chosen reference. This is done by minimizing

$$
\sum_{\mathrm{i}}\left\|\left[\begin{array}{c}
\bar{q}_{x}^{\mathrm{i}} \\
\bar{q}_{y}^{\mathrm{i}}
\end{array}\right]-\left[\begin{array}{ll}
c_{x x} & c_{x y} \\
c_{y x} & c_{y y}
\end{array}\right] \cdot\left[\begin{array}{c}
q_{x}^{\mathrm{i}} \\
q_{y}^{\mathrm{i}}
\end{array}\right]\right\|^{2},
$$

where $\mathbf{q}^{\mathrm{i}}=\left(q_{x}^{\mathrm{i}}, q_{y}^{\mathrm{i}}\right)$ are the detected peak positions in the diffraction patterns and $\overline{\mathbf{q}}^{\mathrm{i}}=\left(\bar{q}_{x}^{\mathrm{i}}, \bar{q}_{y}^{\mathrm{i}}\right)$ are the ones for the reference pattern. This allows us to immediately obtain the strain components

$$
\begin{aligned}
& \varepsilon_{x x}=c_{x x}-1, \quad \varepsilon_{y y}=c_{y y}-1, \\
& \varepsilon_{x y}=\left(c_{x y}+c_{y x}\right) / 2, \quad \omega=\left(c_{x y}-c_{y x}\right) / 2 .
\end{aligned}
$$

Obviously, the reference pattern also contains noise and any error in its analysis impacts the performance of the technique since the fitting would determine the transformation necessary to adapt each diffraction pattern to an inaccurate reference. To reduce this effect, we have averaged the positions extracted from several patterns, recorded in a region which is supposed to be free of strain. The resulting code is freely available under a GPLv3 License. ${ }^{25,26}$

When applied to the simulated diffraction patterns, we get the results shown in Fig. $3(\mathrm{c})$. We can see immediately that the input strain is recovered with good accuracy $\left(\Delta<2 \times 10^{-3}\right)$ and precision $\left(\sigma=4.6 \times 10^{-4}\right)$ although the value is slightly too low. The performance in the simulations appears between those of NBED $(\sigma=11$ $\left.\times 10^{-4}\right)$ and N-PED $\left(\sigma=2 \times 10^{-4}\right){ }^{12}$

We implemented this idea experimentally by fabricating an annular aperture, which, placed in the illumination system of a TEM, realizes the desired hollow-cone illumination. The illumination lenses of the TEM focus the electron beam on the sample, generating a fine electron probe with a wave function given by the aperture's Fourier transform. Alternatively, the aperture constitutes the momentum spectrum of the impinging beam, and hence, each point of the ring in the aperture corresponds to a tilted impinging plane wave. Such an aperture with a diameter of $20 \mu \mathrm{m}$ and an annulus width of $0.9 \mu \mathrm{m}$ was manufactured by milling a $2 \mu \mathrm{m}$ thick gold film with the focused ion beam of a dual beam instrument and inserted in a probe-corrected $\operatorname{Titan}^{3}$ operated at $300 \mathrm{kV}$. The semiconvergence angle $\alpha$ needs to be chosen carefully, as it impacts greatly the obtained result. If $\alpha$ is too low (far below the Bragg angle $\theta_{B}$ of the lowest-order excited reflection), the spatial resolution will be compromised. If $\alpha$ is much higher than $\theta_{B}$, the very large number of overlapping rings makes the patterns difficult to analyze. We have found that values of $\alpha$ slightly below or equal to $2 \times \theta_{B}$ provide a good compromise between the spatial resolution and the overlap. In this case, we have used $\alpha=5.9 \mathrm{mrad}$. The semiconvergence angle is widely tunable on any microscope with an illumination system made of three condenser lenses and it can also be
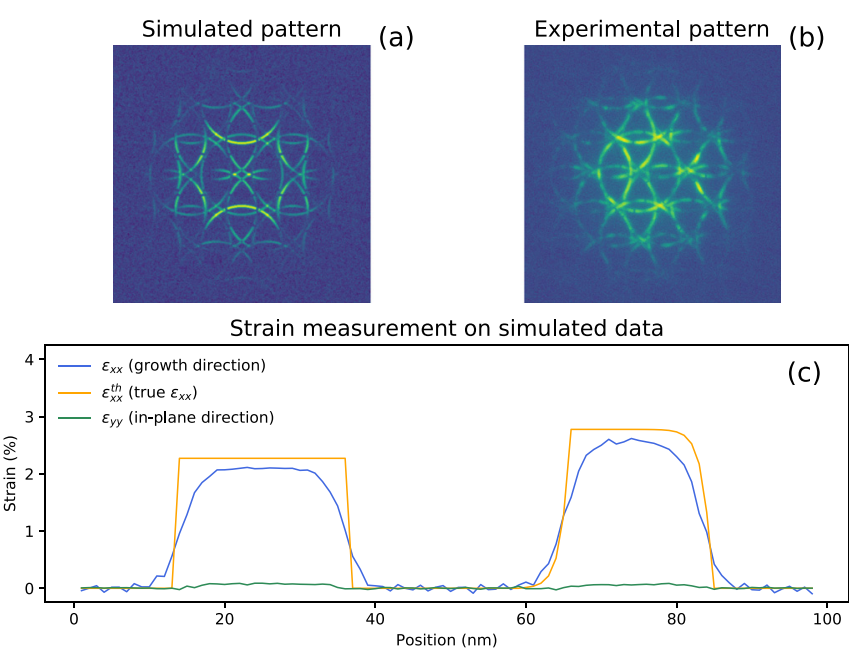

FIG. 3. Simulated strain measurement. Bessel beam diffraction patterns have been simulated (a) to reproduce experimental parameters and conditions (b). These patterns have been analyzed using our in-house developed algorithm to assess the ability of this approach to effectively detect strain (c). The results show that the approach is accurate, though noisy. 
tuned, with a few limits, on other microscopes, although in the last case, larger variations may require a further aperture replacement.

Since this microscope is not fitted with an image corrector, a direct observation of the probe shape might be unreliable, and the expected resolution is best estimated on theoretical grounds and then compared with the sharpness observed in the experimental features. For a conical illumination from an infinitely thin annular aperture, the probe shape is described by a Bessel auxiliary function $J_{0}\left(\alpha k_{0} r_{\perp}\right)$ although in a realistic situation with a finite-width annulus, this is only an approximation and the probe shape needs to be simulated numerically. $^{27,28}$

For conventional nonaberrated electron probes, the wave function is described by an Airy disk, and the resolution is assessed through the full width at half maximum of its probability distribution. In previous experimental demonstrations of strain retrieval with NBED or N-PED, the resolution has been estimated to be $\approx 0.9 \mathrm{~nm} .{ }^{11,29}$ However, in the experimental conditions used ( $\alpha=5.9 \mathrm{mrad}$ ), the simulated probe profile has a full width at half maximum of $70 \mathrm{pm}$, which is not a good measure of resolution owing to the long (though weak) "tails" of the intensity distribution. ${ }^{27,28,30}$ We therefore chose to estimate the resolution by comparing the sharpness of features between high angle annular dark field STEM (HAADF-STEM) images acquired with a conventional high-resolution probe and with our modified beam (see the supplementary material) obtaining a resolution of $1.3 \mathrm{~nm}$. The width of these beams also depends heavily on the width of the annular slit, and a better resolution is likely possible (see the supplementary material). These approximate electron Bessel beams are expected to be resistant to spherical aberrations, ${ }^{27,30}$ and the performance demonstrated here could potentially be reproducible in older nonaberrationcorrected TEMs. While distortions in the projection systems can potentially affect the recorded pattern, their impact on the measured strain is negligible as long as this is measured relative to a reference region. ${ }^{31}$

Using this setup, we test our proposed method on a well-known and characterized sample: a stack of $\mathrm{Si} / \mathrm{SiGe}$ layers grown on a silicon substrate. ${ }^{10,11,29}$ We scanned the beam in a linear fashion using a conventional charge coupled device camera to record one diffraction pattern for each beam position. By using the sample morphology as observed from the microscopy images, as well as the concentration of $\mathrm{Ge}$ in the SiGe layers as measured by secondary ion mass spectroscopy, ${ }^{32}$ we also performed a finite element modeling (FEM) simulation that can be compared with the experimental data as shown in Fig. 4(b). For a more accurate assessment of the resolution, the simulated strain has been convoluted with the simulated probe intensity. By measuring the root-mean-square value of strain in the first part where no strain is expected, we can assess the noise level and hence the strain precision of the technique, to be better than $\sigma=2.5 \times 10^{-4}$. The match with the simulated strain appears very accurate. Also shown is a strain measurement acquired by N-PED on a lamella of the same sample and previously published by Rouvière et al. ${ }^{11}$ This measurement is used here to represent the state of the art.

The difference between the three plots appears small. Figure 4(c) shows that the match between experiment and simulations, assessed in the regions between the layers, is $1.5 \times 10^{-3}$ or better confirming the good accuracy and appears close to the performance of N-PED. The difference at the layer's edges is presumably due to imperfections in our hypothesized probe shape, while the peak height is not considered as a measure of accuracy as it likely suffers from imperfections in

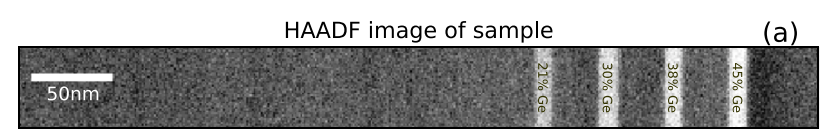

Strain measurement on Si/SiGe multiplayer
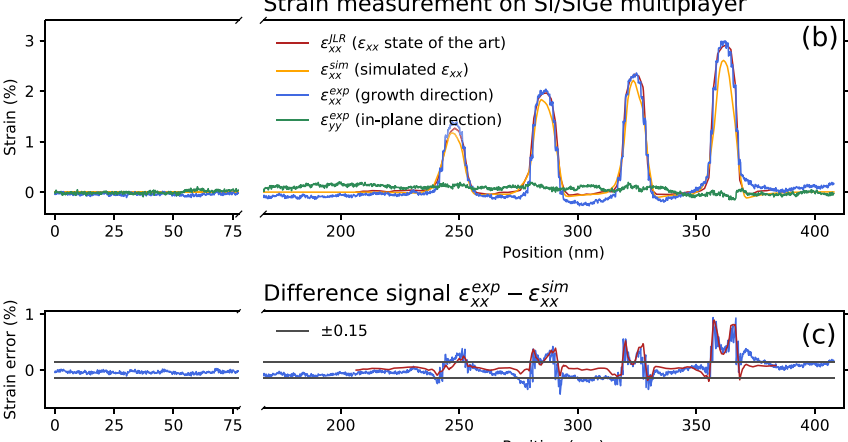

Difference signal $\varepsilon_{x x}^{\text {exp }}-\varepsilon_{x x}^{\text {sim }}$

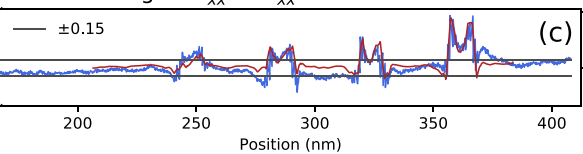

FIG. 4. Experimental measure of strain. (a) Scanning transmission electron microscopy (STEM) image of the multilayer sample. (b) Strain recovered from the experimental data, compared with a finite element simulation of the strain profile and with the N-PED measurement from the study by Rouviére et al. ${ }^{11}$ (c) Difference between measured and simulated strain. The difference is below $1.5 \times 10^{-3}$, except at the interface where it is likely a matter of resolution/probe shape. The red curve, consisting of only 96 points, appears deceptively smoother.

SIMS data used in the FEM modeling, and a similar deviation is observed in both N-PED and Bessel diffraction.

With this comparison, we show how Bessel beam diffraction can detect strain with performances (precision $2.5 \times 10^{-3}$, accuracy $1.5 \times 10^{-3}$ ) which appear superior to those reported for NBED and approach those of N-PED. While Bessel beam diffraction cannot cover many other use-cases supported by PED (e.g., orientation mapping or determination of complex crystal structures), it does not require expensive specialized hardware and can be potentially implemented in any current generation TEM with minimal instrumental modifications (and thus disruptions) and downtime. Furthermore, the technique requires no further alignment beyond a standard STEM experiment and the analysis code is freely available, ${ }^{25,26}$ making it experimentally very accessible and an interesting approach for the study of strain.

See the supplementary material for methodology information on simulations (multislice and finite element) and data processing. Also included are numerical simulations of intensity distributions for different types of electron probes (Fig. S1) and an experimental test of the resolution comparing the Bessel beams with conventional high resolution probes (Fig. S2).

G.G. acknowledges support from a postdoctoral fellowship Grant from the Fonds Wetenschappelijk Onderzoek - Vlaanderen (FWO). A.B. and J.V. acknowledge support from the FWO under the Project No. G.0934.17N "Compressed sensing enabling low dose imaging in transmission electron microscopy". C.M. acknowledges support from the Deutsche Forschungsgemeinschaft (DFG) under Contract No. RO2057/12-2 within the research unit FOR2213. We gratefully thank the CEA-LETI, Grenoble, for providing us with the sample. 


\section{REFERENCES}

${ }^{1}$ J. Cressler, Silicon Heterostructure Devices (CRC Press, 2007).

${ }^{2}$ M. Chu, Y. Sun, U. Aghoram, and S. E. Thompson, Annu. Rev. Mater. Res. 39, 203 (2009).

${ }^{3}$ C. Auth, A. Cappellani, J.-S. Chun, A. Dalis, A. Davis, T. Ghani, G. Glass, T. Glassman, M. Harper, M. Hattendorf, P. Hentges, S. Jaloviar, S. Joshi, J. Klaus, K. Kuhn, D. Lavric, M. Lu, H. Mariappan, K. Mistry, B. Norris, N. RahhalOrabi, P. Ranade, J. Sandford, L. Shifren, V. Souw, K. Tone, F. Tambwe, A. Thompson, D. Towner, T. Troeger, P. Vandervoorn, C. Wallace, J. Wiedemer, and C. Wiegand, in 2008 Symposium on VLSI Technology (IEEE, 2008), Vol. 12, pp. 128-129.

${ }^{4}$ V. Senez, A. Armigliato, I. De Wolf, G. Carnevale, R. Balboni, S. Frabboni, and A. Benedett, J. Appl. Phys. 94, 5574 (2003).

${ }^{5}$ G. Xiong, O. Moutanabbir, M. Reiche, R. Harder, and I. Robinson, Adv. Mater. 26, 7747 (2014).

${ }^{6}$ A. Béché, J. Rouvière, J. Barnes, and D. Cooper, Ultramicroscopy 131, 10 (2013).

${ }^{7}$ S. Kim, S. Lee, Y. Oshima, Y. Kondo, E. Okunishi, N. Endo, J. Jung, G. Byun, S. Lee, and K. Lee, Appl. Phys. Lett. 102, 161604 (2013).

${ }^{8}$ A. Ishizuka, M. Hytch, and K. Ishizuka, J. Electron Microsc. 66, 217 (2017).

${ }^{9}$ A. Pofelski, S. Woo, B. Le, X. Liu, S. Zhao, Z. Mi, S. Löffler, and G. Botton, Ultramicroscopy 187, 1 (2018).

${ }^{10}$ D. Cooper, T. Denneulin, N. Bernier, A. Béché, and J. L. Rouvière, Micron 80, 145 (2016).

"1].-L. Rouviere, A. Béché, Y. Martin, T. Denneulin, and D. Cooper, Appl. Phys. Lett. 103, 241913 (2013).

${ }^{12}$ C. Mahr, K. Müller-Caspary, T. Grieb, M. Schowalter, T. Mehrtens, F. F. Krause, D. Zillmann, and A. Rosenauer, Ultramicroscopy 158, 38 (2015).

${ }^{13} \mathrm{~K}$. Müller, A. Rosenauer, M. Schowalter, J. Zweck, R. Fritz, and K. Volz, Microsc. Microanal. 18, 995 (2012).

${ }^{14}$ A. S. Eggeman and P. A. Midgley, Adv. Imaging Electron Phys. 170, 1 (2012).

${ }^{15}$ J. P. Morniroli, Large-Angle Convergent-Beam Electron Diffraction (LACBED): Applications to Crystal Defects (Société Française des Microscopies, 2002), p. 431 .
${ }^{16}$ J. P. Morniroli, F. Houdellier, C. Roucau, J. Puiggalí, S. Gestí, and A. Redjaïmia, Ultramicroscopy 108, 100 (2008).

${ }^{17}$ J.-L. Rouviere, "Method to facilitate positioning of diffraction spots," U.S. patent 2013/0206968 A1 (2011).

${ }^{18}$ A. Rosenauer and M. Schowalter, Microscopy of Semiconducting Materials 2007 (Springer Netherlands, Dordrecht, 2008), pp. 170-172.

${ }^{19}$ D. Van Dyck, Ultramicroscopy 109, 677 (2009).

${ }^{20}$ E. Prestat, "Quantitative TEM and STEM study of Pt-nanoparticles coarsening and $\mathrm{Ge}(\mathrm{Mn})$-based ferromagnetic nanostructures," Ph.D. thesis (Karlsruher Institut für Technologie and Université de Grenoble, 2013).

${ }^{21}$ F. F. Krause, K. Müller, D. Zillmann, J. Jansen, M. Schowalter, and A. Rosenauer, Ultramicroscopy 134, 94 (2013).

${ }^{22}$ M. Schowalter, A. Rosenauer, J. T. Titantah, and D. Lamoen, Acta Crystallogr., Sect. A 65, 5 (2009).

${ }^{23}$ K. Muraki, S. Fukatsu, Y. Shiraki, and R. Ito, Appl. Phys. Lett. 61, 557 (1992).

${ }^{24}$ T. Grieb, F. F. Krause, C. Mahr, D. Zillmann, K. Müller-Caspary, M. Schowalter, and A. Rosenauer, Ultramicroscopy 181, 50 (2017).

${ }^{25}$ W. Ghielens, G. Guzzinati, and Toon Calders, see https://bitbucket.org/ lutosensis/tem-thesis/ for "Analysis Code is Published on BitBucket" (2018).

${ }^{26}$ G. Guzzinati, W. Ghielens, C. Mahr, A. Béché, A. Rosenauer, T. Calders, and J. Verbeeck, Electron Bessel beam diffraction patterns, line scan of $\mathrm{Si} / \mathrm{SiGe}$ multilayer (Version v1) [Data set]. Zenodo (2019).

${ }^{27}$ V. Grillo, J. Harris, G. C. Gazzadi, R. Balboni, E. Mafakheri, M. R. Dennis, S. Frabboni, R. W. Boyd, and E. Karimi, Ultramicroscopy 166, 48 (2016).

${ }^{28}$ K. Saitoh, K. Hirakawa, H. Nambu, N. Tanaka, and M. Uchida, J. Phys. Soc. Jpn. 85, 043501 (2016).

${ }^{29}$ A. Béché, J. L. Rouvière, L. Clément, and J. M. Hartmann, Appl. Phys. Lett. 95, 123114 (2009).

${ }^{30}$ V. Grillo, E. Karimi, G. C. Gazzadi, S. Frabboni, M. R. Dennis, and R. W. Boyd, Phys. Rev. X 4, 011013 (2014).

${ }^{31}$ C. Mahr, K. Müller-Caspary, R. Ritz, M. Simson, T. Grieb, M. Schowalter, F. F. Krause, A. Lackmann, H. Soltau, A. Wittstock, and A. Rosenauer, Ultramicroscopy 196, 74 (2019).

${ }^{32}$ A. Béché, J. L. Rouvière, J. P. Barnes, and D. Cooper, Ultramicroscopy 111, 227 (2011). 